SISSA $1 / 98 / \mathrm{FM}$

physics/9801020

\title{
Finite Mass Effect on Two Photon Processes in Hydrogenic Systems: Effective Scalar Photon Interaction
}

\author{
C. K. Au ${ }^{1,3}$ and C. S. Chu ${ }^{2,3}$ \\ 1 Department of Physics and Astronomy, University of South Carolina, Columbia, SC \\ 29208, USA \\ 2 International School for Advanced Studies (SISSA), Via Beirut 2, 34014 Trieste, Italy \\ 3 Department of Physics, The Chinese University of Hong Kong, Hong Kong, China \\ email: au@sc.edu,cschu@tao.fm.sissa.it
}

\begin{abstract}
We consider a hydrogenic system with a nucleus of finite mass. The coupling of the radiation field to the center of mass motion gives rise to an effective scalar type coupling. This induced scalar photon interaction emerges as a correction in competition with the usual multipole interactions. This effect is particularly important in positronium where the electric quadrupole interaction is totally suppressed. We illustrate this effect with the two-photon decay of metastable hydrogenic systems.
\end{abstract}




\section{Introduction}

Recently, one of us [1] pointed out, within the framework of nonrelativistic spinless QED, the emergence of an effective scalar photon interaction for the atomic (hydrogenic) internal degree of freedom due to finite mass corrections. In this simple model, the atom is a composite system with two particles of finite masses $\left(m_{1}\right.$ and $\left.m_{2}\right)$ and charges $\left(Z_{1}\right.$ and $Z_{2}$ ) of opposite sign. In contrast to the relative motion which couples to the radiation field vectorially through minimal coupling, the coupling of the center of mass motion to the radiation field induces an effective scalar type coupling for the relative motion. Thus, when finite nuclear mass effect is taken into account, the induced scalar photon interaction leads to a modification of the multipole interactions of the atom with radiation.

Consider a system consisting of two particles of masses $m_{1}, m_{2}$ and charges $Z_{1} e, Z_{2} e$. Let $\mathbf{R}$ and $\mathbf{r}$ denote the center of mass (c.m.) and the relative coordinate vectors and $\mathbf{P}$ and $\mathbf{p}$ denote the momenta conjugate to $\mathbf{R}$ and $\mathbf{r}$. To be definite, we refer to particle 1 as the nucleus and the particle 2 as the electron. The finite mass correction is characterized by the dimensionless quantity

$$
\delta=m_{2} / M, \quad \text { where } M=m_{1}+m_{2}
$$

is the total mass of the system. In general, $0 \leq \delta \leq 1$. The infinite nuclear mass limit corresponds to $\delta=0$. It has been shown [1] that the spinless non-relativistic QED Hamiltonian describing this system is given by

$$
H=H_{0}+H_{\text {int }}
$$

where

$$
\begin{aligned}
& H_{0}=H_{2 p a r t i c l e}+H_{\text {rad }}, \\
& H_{\text {int }}=H_{V}+H_{S}+H_{s g} .
\end{aligned}
$$

$H_{0}$ is the Hamiltonian describing the two-particle system $\left(H_{2 p a r t i c l e}\right)$ and the radiation field $\left(H_{\text {rad }}\right) . H_{\text {int }}$ describes the interaction between the two-particle system and the radiation field. $H_{V}$ describes the coupling of the radiation to the relative motion and is given by the usual vector photon type interaction. $H_{s g}$ is a seagull type interaction responsible for simultaneous two-photon processes. $H_{S}$ describes the coupling of the radiation to the c.m. and is given by an effective scalar photon interaction Hamiltonian. Explicitly, the forms of $H_{V}, H_{S}$, and $H_{s g}$ are [1]:

$$
H_{V}=\sum_{(\epsilon, \mathbf{k})} \sqrt{\frac{2 \pi}{\omega}} e \mathbf{p} \cdot \epsilon e^{i \mathbf{k} \cdot \mathbf{R}}\left(\frac{Z_{2}}{m_{2}} e^{i(\delta-1) \mathbf{k} \cdot \mathbf{r}}-\frac{Z_{1}}{m_{1}} e^{i \delta \mathbf{k} \cdot \mathbf{r}}\right) a_{(\epsilon, \mathbf{k})}+h . c .
$$




$$
\begin{aligned}
& H_{S}=-\sum_{(\epsilon, \mathbf{k})} \sqrt{\frac{2 \pi}{\omega}} \frac{e \mathbf{P} \cdot \epsilon}{M} e^{i \mathbf{k} \cdot \mathbf{R}}\left(Z_{1} e^{i \delta \mathbf{k} \cdot \mathbf{r}}+Z_{2} e^{i(\delta-1) \mathbf{k} \cdot \mathbf{r}}\right) a_{(\epsilon, \mathbf{k})}+h . c . \\
& H_{s g}=\frac{Z_{1}^{2} e^{2}}{2 m_{1}} \mathbf{A}\left(\mathbf{r}_{1}\right)^{2}+\frac{Z_{2}^{2} e^{2}}{2 m_{2}} \mathbf{A}\left(\mathbf{r}_{2}\right)^{2}
\end{aligned}
$$

where

$$
\mathbf{A}(\mathbf{r})=\sum_{(\epsilon, \mathbf{k})} \epsilon \sqrt{\frac{2 \pi}{\omega}}\left[e^{i \mathbf{k} \cdot \mathbf{r}} a_{(\epsilon, \mathbf{k})}+\text { h.c. }\right],
$$

and $\epsilon$ is the polarization vector and $a_{(\epsilon, \mathbf{k})}$ is the annhiliation operator for the photon.

It has long been accepted that within nonrelativistic QED, a single photon transition between two $S$-states $(L=0)$ is impossible [2]. This readily follows from parity consideration if only the vector interaction is assumed. In [1], the effects of finite mass correction on one-photon transitions were studied. Taking into account the induced scalar interaction, it was shown that the metastable $2 S$ state can decay by one-photon emission, which is otherwise forbidden if the nucleus is taken to be infinitely massive. Being a finite mass correction, this one photon decay rate is generally much smaller than the two photon decay rate. The M1 decay [3], though allowed relativisticaly, is not considered here.

For a self conjugate system with $m_{1}=m_{2}$ and $Z_{1}=-Z_{2}$, it turns out that this finite mass correction effect vanishes in the one-photon decay channel and so the leading effect of finite mass correction will appear in two-photon processes. It is the aim of this report to provide a systematic study of finite mass effects on two photon processes.

We now give an estimate of the role of the effective scalar photon interaction. For simplicity, we ignore the overall change in the state of the center of mass motion, bearing in mind that a nonvanishing effective scalar photon interaction requires a nonzero velocity for the center of mass motion. But in the calculations reported in sections 2 and 3 below, we do include the effects of the c.m. recoil. As an example, we consider the two-photon decay of positronium from the metastable $\mid 2 S>$ state to $\mid 1 S>$ state. In this case, $Z_{1}=-Z_{2}=1, m_{1}=m_{2}=m_{e}, \delta=1 / 2$ and $M=2 m_{e}$. On expanding the exponential factors $\exp (-i \mathbf{k} \cdot \mathbf{r} / 2)$ and $\exp (-i \mathbf{k} \cdot \mathbf{r} / 2)$ in both eqns. (4) and (5), we readily observe that all even electric multipole interactions are totally suppressed in positronium [四]. We now focus on the dominant decay channels. For the E1 (electric dipole) channel arising from the vector interaction in (四), the net result as compared to the massive hydrogenic case is that the electronic mass is replaced by the reduced mass in positronium (a difference by a factor of 2). Since the E2 channel is totally suppressed, the next contribution comes from the E3 channel giving rise to a correction to order $\alpha^{4}$ ( $\alpha$ being the fine structure constant) to the leading 2E1 decay amplitude. In contrast, on expanding the factor $e^{i \mathbf{k} \cdot \mathbf{r} / 2}-e^{i \mathbf{k} \cdot \mathbf{r} / 2}$ in the effective scalar photon interaction in (5), we see that the leading nonzero interaction 
becomes

$$
H_{S} \sim H_{S}^{(1)} \equiv-\sum_{(\epsilon, \mathbf{k})} \sqrt{\frac{2 \pi}{\omega}} \cdot \frac{e \mathbf{P} \cdot \epsilon}{2 m_{e}} e^{i \mathbf{k} \cdot \mathbf{R}}(i \mathbf{k} \cdot \mathbf{r}) a_{(\epsilon, \mathbf{k})}+h . c .
$$

We notice that as far as the atomic coordinate is concerned, the interaction in (8) behaves like an E1 (electric dipole) interaction whose coupling strength depends on the center of mass velocity. For comparsion, we also approximate the vector interaction $H_{V}$ of (四) by its leading E1 interaction:

$$
H_{V} \sim H_{V}^{(1)} \equiv-\sum_{(\epsilon, \mathbf{k})} \sqrt{\frac{2 \pi}{\omega}} \cdot \frac{e \mathbf{p} \cdot \epsilon}{m_{e} / 2} e^{i \mathbf{k} \cdot \mathbf{R}} a_{(\epsilon, \mathbf{k})}+h . c .
$$

Both interactions $H_{S}^{(1)}$ and $H_{V}^{(1)}$ couple to the $\mid n P>$ state, but $H_{S}^{(1)}$ has a nonzero coupling to the $\mid 2 P>$ state whereas $H_{V}^{(1)}$ does not because of the $\mid 2 S>$ and $\mid 2 P>$ degeneracy. Thus the $\mid 2 P>$ state contributes to the two scalar photon decay of metastable postitronium, but not to the two vector photon decay nor to the one vector-one scalar photon decay. This follows from the trivial identity

$$
<n P|\mathbf{p}| 2 S>=\frac{i m_{e}}{2} E_{n 2}<n P|\mathbf{r}| n S>,
$$

where $E_{n 2}$ is the energy difference between the $\mid n P>$ and $\mid 2 S>$ positronium states and we have explicitly used the fact that the reduced mass in positronium is $m_{e} / 2$. Thus we see that the contribution of $H_{S}^{(1)}$ to the two photon decay amplitude relative to that of $H_{V}^{(1)}$ is of the order

$$
\frac{<H_{S}^{(1)}>}{<H_{V}^{(1)}>} \sim \frac{1}{4} \frac{k P}{m_{e} E_{n 2}} \sim \frac{P}{m_{e}} \sim \frac{v}{c}
$$

where $v$ is the velocity of the positronium center of mass motion, and we have explicitly reinserted $c$. This simple analysis leads us to conclude that

$$
\frac{M_{S S}}{M_{S V}} \sim \frac{M_{V S}}{M_{V V}} \sim \frac{v}{c} .
$$

In this brief report, we consider a general hydrogenic system and study the effect of the scalar photon interaction $H_{S}$ on the two-photon transition between two $S$-states $(n S$ to $n^{\prime} S$ ). Thus, the general matrix element is of the form

$$
\begin{aligned}
& <n^{\prime} S\left|H_{s g}\right| n S>\quad \text { or } \\
& <n^{\prime} S\left|H_{i} G H_{j}\right| n S>, \quad \text { where } H_{i(j)} \text { can be either } H_{V} \text { or } H_{S},
\end{aligned}
$$

and $G$ is the hydrogenic Coluomb Green's function. For example, $\left\langle n^{\prime} S\left|H_{V} G H_{V}\right| n S\right\rangle$ is the usual two vector photon transition matrix element between $\mid n S>$ and $\mid n^{\prime} S>$. This 
can be calculated in closed form, following a method used by Gavrila and Costescu [5]. More interesting are matrix elements of the type

$$
\begin{aligned}
& <n^{\prime} S\left|H_{S} G H_{V}\right| n S>, \quad \text { the mixed vecor-scalar matrix element, } \\
& <n^{\prime} S\left|H_{S} G H_{S}\right| n S>, \quad \text { the two scalar photon transition matrix element. }
\end{aligned}
$$

The two scalar photon transtion matrix element for the special case where $\mid n S>$ and $\mid n^{\prime} S>$ are both the $\mid 1 S>$ ground state has in fact been evaluated analytically by one of us [6]. It is interesting to point out that in the calculation reported in [6], the two scalar photon transition matrix element was only used as a calculational tool and no physical significance was attached to it. The generalization to arbitrary $n$ and $n^{\prime}$ can be easily carried out and the result will be reported in section 2 below. The mixed scalar-vector two photon transition matrix element has never been studied before. It can also be evaluated in closed form and will be reported in section 2 below. In section 3, we apply these results to study the two-photon decay of the metastable $2 S$ state. The angular distribution of the two-photon decay spectrum is reported.

\section{Exact two photon matrix elements}

We will see in the following that for a scattering process 1 from $(\epsilon, \mathbf{k})$ to $\left(\epsilon^{\prime}, \mathbf{k}^{\prime}\right)$, it is useful to introduce the the following two photon matrix elements:

the two vector photon matrix element,

$$
\begin{array}{r}
M_{V V}\left(\Omega, \mathbf{k}_{2}, \mathbf{k}_{1}\right) \equiv \iint d \mathbf{r}_{1} d \mathbf{r}_{2} \epsilon^{\prime} \cdot \mathbf{p} u_{n^{\prime} S}\left(\mathbf{r}_{2}\right) e^{-i \mathbf{k}_{2} \cdot \mathbf{r}_{2}} G\left(\mathbf{r}_{2}, \mathbf{r}_{1}, \Omega\right) e^{i \mathbf{k}_{1} \cdot \mathbf{r}_{1}} \epsilon \cdot \mathbf{p} u_{n S}\left(\mathbf{r}_{1}\right) \\
=\iint d \mathbf{p}_{1} d \mathbf{p}_{2} \epsilon^{\prime} \cdot \mathbf{p}_{2} u_{n^{\prime} S}\left(\mathbf{p}_{2}-\mathbf{k}_{2}\right) G\left(\mathbf{p}_{2}, \mathbf{p}_{1}, \Omega\right) \epsilon \cdot \mathbf{p}_{1} u_{n S}\left(\mathbf{p}_{1}-\mathbf{k}_{1}\right)
\end{array}
$$

the two scalar photon transition matrix element

$$
M_{S S}\left(\Omega, \mathbf{k}_{2}, \mathbf{k}_{1}\right) \equiv \iint d \mathbf{p}_{1} d \mathbf{p}_{2} u_{n^{\prime} S}\left(\mathbf{p}_{2}-\mathbf{k}_{2}\right) G\left(\mathbf{p}_{2}, \mathbf{p}_{1}, \Omega\right) u_{n S}\left(\mathbf{p}_{1}-\mathbf{k}_{1}\right)
$$

and the mixed scalar-vector two photon transition matrix element becomes

$$
\begin{aligned}
& M_{S V}\left(\Omega, \mathbf{k}_{2}, \mathbf{k}_{1}\right) \equiv \iint d \mathbf{p}_{1} d \mathbf{p}_{2} u_{n^{\prime} S}\left(\mathbf{p}_{2}-\mathbf{k}_{2}\right) G\left(\mathbf{p}_{2}, \mathbf{p}_{1}, \Omega\right) \epsilon \cdot \mathbf{p}_{1} u_{n S}\left(\mathbf{p}_{1}-\mathbf{k}_{1}\right) \\
& M_{V S}\left(\Omega, \mathbf{k}_{2}, \mathbf{k}_{1}\right) \equiv \iint d \mathbf{p}_{1} d \mathbf{p}_{2} \epsilon^{\prime} \cdot \mathbf{p}_{2} u_{n^{\prime} S}\left(\mathbf{p}_{2}-\mathbf{k}_{2}\right) G\left(\mathbf{p}_{2}, \mathbf{p}_{1}, \Omega\right) u_{n S}\left(\mathbf{p}_{1}-\mathbf{k}_{1}\right)
\end{aligned}
$$

\footnotetext{
${ }^{1}$ Notice that for a two photon decay process, one only need to replace $\mathbf{k}$ by $-\mathbf{k}$ and $\omega$ by $-\omega$ in (15) 18$)$.
} 
Here $G\left(\mathbf{p}_{2}, \mathbf{p}_{1}, \Omega\right)$ is the Coulomb Green's function $\left(\Omega-H_{0}\right)^{-1}$ in momentum representation, $\mathbf{k}_{1}=-\delta \mathbf{k}$ or $(1-\delta) \mathbf{k}$ and $\mathbf{k}_{2}=\delta \mathbf{k}^{\prime}$ or $-(1-\delta) \mathbf{k}^{\prime}$ originating from the finite mass effect.

To simplify the discussion, we limt ourself to a hydrogenic atom where an electron is bound to a positive charge $+Z|e|$. We take advantage of the fact that the $\mid n S>$ wave function can be generated from the $\mid 1 S>$ wave function by a parametric differentiation:

$$
\psi_{n S}(p)=-\left.\frac{1}{n^{7 / 2} n !}\left(\frac{8 \lambda^{5}}{\pi^{2}}\right)^{1 / 2} B_{n}\left(-\frac{2 \lambda}{n} \partial_{\beta}\right) \frac{1}{\left(p^{2}+\beta^{2}\right)^{2}}\right|_{\beta=\lambda / n}
$$

where

$$
B_{n}(x) \equiv L_{n}^{1}(x)-2 L_{n}^{2}(x), \quad B_{1}(x)=1,
$$

and $L_{\mu}^{\nu}(x)$ are the associated Laguerre functions. In (19), $\beta, n, \lambda$ are independent variables and

$$
\lambda \equiv \alpha Z \mu \text {. }
$$

Here $\mu$ is the reduced mass of the electron in the atom. We also use natural units where $c=\hbar=1$.

Next we used the fact [5] that the integral

$$
I\left(\Omega, \mathbf{k}_{2}, \mathbf{k}_{1}\right) \equiv \iint d \mathbf{p}_{1} d \mathbf{p}_{2}\left(\left(\mathbf{p}_{2}-\mathbf{k}_{2}\right)^{2}+\eta^{2}\right)^{-1} G\left(\mathbf{p}_{2}, \mathbf{p}_{1}, \Omega\right)\left(\left(\mathbf{p}_{1}-\mathbf{k}_{1}\right)^{2}+\beta^{2}\right)^{-1}
$$

can be evaluated analytically in closed form. The analytical form of $I$ is given in (31) below. Then a comparison of (19) and (22), upon noticing the property of tranversality of the photon, clearly suggests that to generate the needed matrix elements, we need the simple relations:

$$
\begin{aligned}
& -\frac{1}{2 \beta} \frac{\partial}{\partial \beta} \frac{1}{\left((\mathbf{p}+\mathbf{k})^{2}+\beta^{2}\right)}=\frac{1}{\left((\mathbf{p}+\mathbf{k})^{2}+\beta^{2}\right)^{2}} \\
& -\frac{1}{4} \epsilon_{i} \frac{\partial}{\partial k_{i}} \frac{1}{\left((\mathbf{p}+\mathbf{k})^{2}+\beta^{2}\right)}=\frac{\epsilon \cdot \mathbf{p}}{\left((\mathbf{p}+\mathbf{k})^{2}+\beta^{2}\right)^{2}} .
\end{aligned}
$$

Thus, the relations expressed in eqs. (23) and (24) become the keys to generate a scalar photon vertex and a vector photon vertex respectively.

Having laid down the rules to obtain the transition matrix elements, we proceed to simply stating the results. We define the following:

$$
\begin{aligned}
& X \equiv i(2 \mu)^{1 / 2}\left(E_{n s}+\Omega\right)^{1 / 2} \\
& E_{n s} \equiv-\lambda^{2} /(2 \mu) n^{2} \\
& \tau \equiv \lambda / X
\end{aligned}
$$




$$
\begin{aligned}
& \beta \equiv \lambda / n, \quad \eta \equiv \lambda / n^{\prime}, \\
& s \equiv \frac{2\left(\gamma^{2} \mathbf{k}^{2}+\beta^{2}-X^{2}\right)\left(\mathbf{k}_{2}^{2}+\eta^{2}-X^{2}\right)-8 X^{2} \mathbf{k}_{1} \cdot \mathbf{k}_{2}}{\left[(X+\beta)^{2}+\mathbf{k}_{1}^{2}\right]\left[(X+\eta)^{2}+\mathbf{k}_{2}^{2}\right]} \\
& p \equiv \frac{\left((X-\beta)^{2}+\mathbf{k}_{1}^{2}\right)\left((X-\eta)^{2}+\mathbf{k}_{2}^{2}\right)}{\left[(X+\beta)^{2}+\mathbf{k}_{1}^{2}\right]\left[(X+\eta)^{2}+\mathbf{k}_{2}^{2}\right]}
\end{aligned}
$$

and

$$
\begin{aligned}
J\left(\Omega, \mathbf{k}_{2}, \mathbf{k}_{1}\right) & \equiv 64 \lambda \mu X\left(\frac{i e^{i \pi \tau}}{2 \sin \pi \tau}\right) \int_{1}^{0_{+}} d \rho \rho^{-\tau} \frac{\left(1-s \rho+p \rho^{2}\right)^{-1}}{\left((X+\beta)^{2}+\mathbf{k}_{1}^{2}\right)\left((X+\eta)^{2}+\mathbf{k}_{2}^{2}\right)} \\
& =\frac{8 \lambda^{5}}{\pi^{2}} I\left(\Omega, \mathbf{k}_{2}, \mathbf{k}_{1}\right)
\end{aligned}
$$

where the integration contour begins at $\rho=1$, runs along the real axis to a point closely on the right of $\rho=0$, encirlces the origin in the counter-clockwise sense and runs back to $\rho=1$. The integral $J$ is expressible in terms of the Apelle type hypergeometric function. The desired matrix elements (15-18) are then expressible in terms of parametric differentiation of $J$. Specifically,

$$
\begin{aligned}
M_{V V} & =\frac{1}{16} \epsilon_{i}^{\prime} \epsilon_{j} \frac{\partial}{\partial k_{2 i}} \frac{\partial}{\partial k_{1 j}} J\left(\Omega, \mathbf{k}_{2}, \mathbf{k}_{1}\right), \\
M_{S S} & =\frac{1}{4} \frac{1}{\beta \eta} \frac{\partial}{\partial \beta} \frac{\partial}{\partial \eta} J\left(\Omega, \mathbf{k}_{2}, \mathbf{k}_{1}\right) \\
M_{S V} & =\frac{1}{8} \frac{1}{\eta} \frac{\partial}{\partial \eta} \epsilon_{i} \frac{\partial}{\partial k_{1 i}} J\left(\Omega, \mathbf{k}_{2}, \mathbf{k}_{1}\right) \\
M_{V S} & =\frac{1}{8} \epsilon_{i}^{\prime} \frac{\partial}{\partial k_{2 i}} \frac{1}{\beta} \frac{\partial}{\partial \beta} J\left(\Omega, \mathbf{k}_{2}, \mathbf{k}_{1}\right) .
\end{aligned}
$$

This completes the analytical calculations of the two-photon transition matrix elements. We would like to point out that the parametric differentiation using $\epsilon_{i} \partial / \partial k_{i}$ or $\epsilon_{i}^{\prime} \partial / \partial k_{i}^{\prime}$ is rather simple upon using the tranversality condition

$$
\epsilon \cdot \mathbf{k}=\epsilon^{\prime} \cdot \mathbf{k}^{\prime}=0
$$

\section{Two photon decay of the metastable $2 S$ state}

In this section, we consider the two photon decay of the metastable $2 S$ state of a hydrogenic system with a electron bound to a positive charge $Z|e|$. We will present all the results consistently up to order $\Delta / M$ and $(Z \alpha)^{2}$, compared to the leading order. 


\subsection{Kinematics}

Let the emitted photons be in the state $(\epsilon, \mathbf{k})$ and $\left(\epsilon^{\prime}, \mathbf{k}^{\prime}\right)$. Without loss of generality, we can take the atom to be initially at rest $(\mathbf{P}=0)$. From the conservation of energy and momentum, we have

$$
\begin{aligned}
& \omega+\omega^{\prime}+\frac{\mathbf{P}^{\prime 2}}{2 M}=\Delta, \\
& \mathbf{P}^{\prime}=-\mathbf{k}-\mathbf{k}^{\prime},
\end{aligned}
$$

where $\Delta=\frac{3}{8} \mu Z^{2} \alpha^{2}$ is the energy difference between the $2 S$ and $1 S$ level. This gives

$$
\omega+\omega^{\prime}+\frac{\omega^{2}+\omega^{\prime 2}+2 \omega \omega^{\prime} \cos \theta}{2 M}=\Delta, \quad \text { where } \cos \theta=\hat{\mathbf{k}} \cdot \hat{\mathbf{k}}^{\prime}
$$

A convenient way to parametrize this equation is to introduce a dimensioness parameter $\sigma$ such that

$$
\begin{aligned}
& \omega=\Delta\left[\sigma+\frac{\Delta \sigma(1-\sigma)}{2 M}(1-\cos \theta)-\frac{\Delta}{2 M} \sigma+o\left(\alpha^{3}\right)\right], \\
& \omega^{\prime}=\Delta\left[(1-\sigma)+\frac{\Delta \sigma(1-\sigma)}{2 M}(1-\cos \theta)-\frac{\Delta}{2 M}(1-\sigma)+o\left(\alpha^{3}\right)\right] .
\end{aligned}
$$

This parametrization satisfies (38) up to order $\Delta / M$. The range of $\omega$ is from 0 to $\Delta-\frac{\Delta^{2}}{2 M}$ and the range of $\sigma$ is from 0 to 1 .

\subsection{Transition amplitudes}

The transition operator is given by

$$
T=H_{1}+H_{s g}+H_{1} \frac{1}{E-H_{0}} H_{1}+\cdots, \quad \text { where } H_{1} \equiv H_{V}+H_{S} .
$$

It is clear that the matrix element for the terms in ellipsis in (40) are of order higher than $(Z \alpha)^{2}$ or $\Delta / M$ and we only need to compute $<f|T| i>$ for the displayed terms in (40).

The initial and final states are

$$
\begin{array}{rll}
|i>=| \mathbf{P}>\otimes & \mid 2 S>\otimes & \mid \text { no photon }>, \\
|f>=| \mathbf{P}^{\prime}>\otimes & \mid 1 S>\otimes & \mid \text { two photons in the modes }(\epsilon, \mathbf{k}),\left(\epsilon^{\prime}, \mathbf{k}^{\prime}\right)>.
\end{array}
$$

It is easy to see that

$$
<f\left|H_{1}\right| i>=0
$$




$$
<f\left|H_{s g}\right| i>=\frac{e^{2}}{m_{2}}\left\{R(1-\delta)+Z^{2} \frac{\delta}{1-\delta} R(\delta)\right\}\left(\epsilon \cdot \epsilon^{\prime}\right) e^{-i\left(\mathbf{k}+\mathbf{k}^{\prime}\right) \cdot \mathbf{R}}
$$

where

$$
R(\delta) \equiv<1 S\left|e^{-i \delta\left(\mathbf{k}+\mathbf{k}^{\prime}\right) \cdot \mathbf{r}}\right| 2 S>,
$$

which to order $(Z \alpha)^{2}$ can be written as

$$
R(\delta)=R_{1}(\delta)+R_{2}(\delta) \cos \theta
$$

where

$$
\begin{aligned}
& R_{1}(\delta)=\frac{256 \sqrt{2}}{729}\left\{\left(\frac{3 \sigma}{8}\right)^{2}+\left(\frac{3(1-\sigma)}{8}\right)^{2}\right\}(Z \alpha)^{2} \delta^{2} \\
& R_{2}(\delta)=\frac{8 \sqrt{2}}{81} \sigma(1-\sigma)(Z \alpha)^{2} \delta^{2}
\end{aligned}
$$

and

$$
<f\left|H_{1} \frac{1}{E-H_{0}} H_{1}\right| i>=e^{-i\left(\mathbf{k}+\mathbf{k}^{\prime}\right) \cdot \mathbf{R}}\left(A\left(\mathbf{k}, \mathbf{k}^{\prime}\right)+B\left(\mathbf{k}, \mathbf{k}^{\prime}\right)+\left(\mathbf{k} \leftrightarrow \mathbf{k}^{\prime}\right)\right),
$$

where $A$ comes from the two vector photon interaction, $B$ comes from the mixed vectorscalar photon interaction. They are:

$$
\begin{aligned}
& A\left(\mathbf{k}, \mathbf{k}^{\prime}\right)=-e^{2}<1 S\left|\mathbf{p} \cdot \epsilon^{\prime}\left(\frac{Z}{m_{2}} e^{i(\delta-1) \mathbf{k}^{\prime} \cdot \mathbf{r}}+\frac{1}{m_{1}} e^{i \delta \mathbf{k}^{\prime} \cdot \mathbf{r}}\right) \hat{G}(\Omega)\left(\frac{Z}{m_{2}} e^{i(\delta-1) \mathbf{k} \cdot \mathbf{r}}+\frac{1}{m_{1}} e^{i \delta \mathbf{k} \cdot \mathbf{r}}\right) \mathbf{p} \cdot \epsilon\right| 2 S> \\
& B\left(\mathbf{k}, \mathbf{k}^{\prime}\right)=-e^{2} \frac{\mathbf{P}^{\prime} \cdot \epsilon}{M}<1 S\left|\left(Z_{1} e^{i \delta \mathbf{k}^{\prime} \cdot \mathbf{r}}+Z_{2} e^{i(\delta-1) \mathbf{k}^{\prime} \cdot \mathbf{r}}\right) \hat{G}(\Omega)\left(\frac{Z}{m_{2}} e^{i(\delta-1) \mathbf{k} \cdot \mathbf{r}}+\frac{1}{m_{1}} e^{i \delta \mathbf{k} \cdot \mathbf{r}}\right) \mathbf{p} \cdot \epsilon\right| 2 S>
\end{aligned}
$$

It is clear that these matrix elements can be reduced to the forms (15) and (18).

In particular, we have (for $n=2, n^{\prime}=1$ ),

$$
\begin{aligned}
M_{V V}= & \frac{32 \sqrt{2} \mu \lambda^{5} X^{3}}{f_{1}^{3} f_{2}^{2}}\left[\left(X^{2}-\beta^{2}+\mathbf{k}_{1}^{2}\right) \frac{F_{A}\left(2-\tau ; 2,2 ; 3-\tau ; x_{1}, x_{2}\right)}{2-\tau}\right. \\
& \left.-\beta y_{1} \frac{F_{A}\left(3-\tau ; 3,3 ; 4-\tau ; x_{1}, x_{2}\right)}{3-\tau}-\beta y_{1} \frac{F_{A}\left(4-\tau ; 3,3 ; 5-\tau ; x_{1}, x_{2}\right)}{4-\tau}\right]\left(\epsilon \cdot \epsilon^{\prime}\right) \\
& +\frac{512 \sqrt{2} \mu \lambda^{5} X^{5}}{f_{1}^{4} f_{2}^{3}}\left[\left(X^{2}-2 \beta^{2}-X \beta+\mathbf{k}_{1}^{2}\right) \frac{F_{A}\left(3-\tau ; 3,3 ; 4-\tau ; x_{1}, x_{2}\right)}{3-\tau}\right. \\
& \left.-\frac{3}{2} \beta y_{1} \frac{F_{A}\left(4-\tau ; 4,4 ; 5-\tau ; x_{1}, x_{2}\right)}{4-\tau}-\frac{3}{2} \beta y_{2} \frac{F_{A}\left(5-\tau ; 4,4 ; 6-\tau ; x_{1}, x_{2}\right)}{5-\tau}\right]\left(\epsilon \cdot \mathbf{k}_{2}\right)\left(\epsilon^{\prime} \cdot \mathbf{k}_{1}\right)
\end{aligned}
$$

and

$$
\begin{aligned}
M_{V S}= & \frac{64 \sqrt{2} \mu \lambda^{4} X^{3}}{f_{1}^{2} f_{2}^{3}}\left(\epsilon \cdot \mathbf{k}_{2}\right)\left[2(X+\lambda)\left(1-\frac{2 \beta(X+\beta)}{f_{1}}\right) \frac{F_{A}\left(2-\tau ; 2,2 ; 3-\tau ; x_{1}, x_{2}\right)}{2-\tau}\right. \\
& +\left(y_{1}^{\prime}+\frac{4 Q_{1} \beta}{f_{1}}\right) \frac{F_{A}\left(3-\tau ; 3,3 ; 4-\tau ; x_{1}, x_{2}\right)}{3-\tau}+\left(y_{2}^{\prime}+\frac{4 Q_{2} \beta}{f_{1}}\right) \frac{F_{A}\left(4-\tau ; 3,3 ; 5-\tau ; x_{1}, x_{2}\right)}{4-\tau} \\
& \left.-\frac{3}{2} \frac{\beta W_{1}}{f_{1}} \frac{F_{A}\left(5-\tau ; 4,4 ; 6-\tau ; x_{1}, x_{2}\right)}{5-\tau}-\frac{3}{2} \frac{\beta W_{2}}{f_{1}} \frac{F_{A}\left(6-\tau ; 4,4 ; 7-\tau ; x_{1}, x_{2}\right)}{6-\tau}\right]
\end{aligned}
$$


where $F_{A}$ is the Apelle's type hypergeometric function,

$$
\begin{aligned}
& \beta=\lambda / 2, \quad \lambda=\alpha Z \mu \\
& X^{2}=2 \mu\left(\left|E_{2 s}\right|+\omega+\frac{\omega^{2}}{2 M}\right) \\
& \tau=\lambda / X, \\
& f_{1}=(X+\beta)^{2}+\mathbf{k}_{1}^{2}, \quad f_{2}=(X+\lambda)^{2}+\mathbf{k}_{2}^{2} \\
& \tilde{f}_{1}=(X-\beta)^{2}+\mathbf{k}_{1}^{2}, \quad \tilde{f}_{2}=(X-\lambda)^{2}+\mathbf{k}_{2}^{2} \\
& g_{1}=\mathbf{k}_{1}^{2}+\beta^{2}-X^{2}, \quad g_{2}=\mathbf{k}_{2}^{2}+\lambda^{2}-X^{2} \\
& y_{1}=\frac{1}{f_{1} f_{2}}\left[-4 g_{2} X\left[(X+\beta)^{2}-\mathbf{k}_{1}^{2}\right]+16 X^{2}(X+\beta)\left(\mathbf{k}_{1} \cdot \mathbf{k}_{2}\right)\right] \\
& y_{2}=\frac{1}{f_{1} f_{2}}\left[4 X\left((X-\lambda)^{2}+\mathbf{k}_{2}^{2}\right)\left(\beta^{2}-X^{2}-\mathbf{k}_{1}^{2}\right)\right] \\
& Q_{1}=\frac{1}{f_{1} f_{2}}\left[-\lambda \beta f_{1} f_{2}+3 \beta(X+\lambda) f_{1} g_{2}+3 \lambda(X+\beta) f_{2} g_{1}-5(X+\beta)(X+\lambda) g_{1} g_{2}\right. \\
& \left.\quad-20 X^{2}(X+\beta)(X+\lambda) \mathbf{k}_{1} \cdot \mathbf{k}_{2}\right] \\
& Q_{2}=\frac{1}{f_{1} f_{2}}\left[-4(X+\beta) \lambda g_{2} \tilde{f}_{1}-4(X+\lambda) \beta g_{1} \tilde{f}_{2}+2 \lambda \beta g_{1} g_{2}+6 \tilde{f}_{1} \tilde{f}_{2}(X+\beta)(X+\lambda)\right] \\
& \quad-\frac{3}{8} y_{1}^{\prime} y_{2}^{\prime}, \\
& s=\frac{1}{f_{1} f_{2}}\left[2 g_{1} g_{2}+8 X^{2} \mathbf{k}_{1} \cdot \mathbf{k}_{2}\right], \quad p=\frac{\tilde{f}_{1} \tilde{f}_{2}}{f_{1} f_{2}} \\
& W_{1}=y_{1} y_{2}^{\prime}+y_{2} y_{1}^{\prime}+s y_{1} y_{1}^{\prime}, \quad W_{2}=y_{2} y_{2}^{\prime}-p y_{1} y_{1}^{\prime}
\end{aligned}
$$

and $x_{1}, x_{2}$ are given by

$$
1-s \rho+p \rho^{2}=\left(1-x_{1} \rho\right)\left(1-x_{2} \rho\right) .
$$

Here $y_{1}^{\prime}, y_{2}^{\prime}$ are obtained from $y_{1}, y_{2}$ by interchanging $\lambda$ with $\beta$ and $\mathbf{k}_{1}$ with $\mathbf{k}_{2}$.

The $2 S$ to $1 S$ amplitude $M_{V V}$ was first evaluated in [7] within the dipole approximation. Higher multipole contributions were calculated in [8]. 2]

One can substitute these expression into (40) and obtain the exact two photon matrix elements. However, to illustrate the finite mass effect, it is enough to do an expansion and keep results up to first order in $\Delta / M$.

\footnotetext{
${ }^{2}$ In (48) above, we have corrected some typos in [8]. In [8], the total power of $f_{1}$ in the first (second) factor of eqn. (7) should be 3 (4) instead of 2 (2); in eqn.(9), it should read $x_{1} * x_{2}$ instead of $x_{1}-x_{2}$; the L.H.S. of eqn. (13) should read $y_{2}$; the L.H.S. of eqn. (14) should read $-y_{1}$. In [8], there was an error (a factor of 2) in one of the terms presented. The correct results are given in (69) and (72) below.
} 
It is convenient to write $<2 S|T| 1 S>$ in the form

$$
<f|T| i>=\frac{\alpha}{m_{2}} \frac{2 \pi}{\sqrt{\omega \omega^{\prime}}} \mathcal{M} .
$$

Using (39) and perform a tedious but systematic and straight forward expansion in power of $Z \alpha$ and $\Delta / M$, one obtain

$$
\mathcal{M}=\left(\hat{\epsilon} \cdot \hat{\epsilon}^{\prime}\right)\left\{g+(Z \alpha)^{2}\left[f+\left(f^{\prime}+f^{\prime \prime} \frac{\mu}{M}\right) \cos \theta\right]\right\}+(Z \alpha)^{2}\left(h^{\prime}+\frac{\mu}{M} h^{\prime \prime}\right)\left(\hat{\epsilon} \cdot \hat{\mathbf{k}}^{\prime}\right)\left(\hat{\epsilon}^{\prime} \cdot \hat{\mathbf{k}}\right),
$$

up to order $(Z \alpha)^{2}$ and first order in $\Delta / M$. We have singled out the $\delta$ dependence and $g, f, f^{\prime}, f^{\prime \prime}$ and $h^{\prime}, h^{\prime \prime}$ are $\delta$ independent. Here $\theta$ is the correlation angle between the two photons in the initial rest frame of the decaying system.

The physical origin of these terms are: $g$ corresponds to the two electric dipole transition without retardation; $f$ corresponds to correction to $g$ to order $(Z \alpha)^{2}$ due to retardation effect; $f^{\prime}, h^{\prime}$ come from the two electric quadrupole transition. $f^{\prime}$ also contains a contribution coming from the seagull term; $f^{\prime \prime}, h^{\prime \prime}$ include the recoil correction to the lowest order in $\Delta / M$.

\subsection{Angular distribution of the decay spectrum}

On substituting $m_{2}$ by $m_{e}$, the decay spectrum is given by

$$
d W=\frac{1}{2 \pi} \frac{\alpha^{2}}{m_{e}^{2}} \sum|\mathcal{M}|^{2} \omega \omega^{\prime} d \omega d \cos \theta .
$$

Using (39), one finds that

$$
\omega \omega^{\prime} d \omega=\Delta^{3} d \sigma \sigma(1-\sigma)\left[1-\frac{\Delta}{2 M}(1+2 \sigma)-\frac{\Delta}{M}(1-\sigma) \cos \theta\right] .
$$

Subsituting $\Delta=\frac{3}{8} \mu(Z \alpha)^{2}=\frac{3}{8}(1-\delta) m_{e}(Z \alpha)^{2}$, one obtain

$$
\begin{aligned}
\frac{d W}{d \sigma d \cos \theta}= & \frac{1}{2 \pi}\left(\frac{3}{8}\right)^{2} Z^{6} m_{e} \alpha^{8}(1-\delta)^{3} . \\
& \sum|\mathcal{M}|^{2} \sigma(1-\sigma) \cdot\left[1-\frac{3}{16}(1+2 \sigma) \frac{\mu}{M}(Z \alpha)^{2}-\frac{3}{8}(1-\sigma) \cos \theta \frac{\mu}{M}(Z \alpha)^{2}\right]
\end{aligned}
$$

where the sum is over the polarizations of the two emitted photons. To second order in $Z \alpha$ and first order in $\Delta / M$, we have

$$
\begin{aligned}
\sum|\mathcal{M}|^{2} & =\left[g^{2}+2 g f(Z \alpha)^{2}\right]\left(1+\cos ^{2} \theta\right)+(Z \alpha)^{2}\left[2 g\left(f^{\prime}-h^{\prime}\right)+2 g\left(f^{\prime \prime}-h^{\prime \prime}\right) \frac{\mu}{M}\right] \cos \theta \\
& +\quad(Z \alpha)^{2}\left[2 g\left(f^{\prime}+h^{\prime}\right)+2 g\left(f^{\prime \prime}+h^{\prime \prime}\right) \frac{\mu}{M}\right] \cos ^{3} \theta
\end{aligned}
$$


Hence, one can write

$$
\begin{aligned}
\frac{d W}{d \cos \theta}=\quad & Z^{6}\left[\left(d_{1}+d_{2}(Z \alpha)^{2}+d_{3} \frac{\mu}{M}(Z \alpha)^{2}\right) \cdot\left(1+\cos ^{2} \theta\right)\right. \\
& \left.+(Z \alpha)^{2}\left(d_{4}+\frac{\mu}{M} d_{5}\right) \cos \theta+(Z \alpha)^{2}\left(d_{6}+\frac{\mu}{M} d_{7}\right) \cos ^{3} \theta\right]
\end{aligned}
$$

with

$$
\begin{array}{ll}
d_{1}=c \int d \sigma \sigma(1-\sigma) g^{2}, & d_{2}=c \int d \sigma \sigma(1-\sigma) 2 g f, \\
d_{3}=-c \int d \sigma 3 / 16 \sigma(1-\sigma)(1+2 \sigma) g^{2}, & d_{4}=c \int d \sigma \sigma(1-\sigma) 2 g\left(f^{\prime}-h^{\prime}\right), \\
d_{5}=c \int d \sigma\left[\sigma(1-\sigma) 2 g\left(f^{\prime \prime}-h^{\prime \prime}\right)-3 / 8 \sigma(1-\sigma)^{2} g^{2}\right], & d_{6}=c \int d \sigma \sigma(1-\sigma) 2 g\left(f^{\prime}+h^{\prime}\right), \\
d_{7}=c \int d \sigma\left[\sigma(1-\sigma) 2 g\left(f^{\prime \prime}+h^{\prime \prime}\right)-3 / 8 \sigma(1-\sigma)^{2} g^{2}\right] &
\end{array}
$$

and $c=\frac{1}{2 \pi}\left(\frac{3}{8}\right)^{2} m_{e} \alpha^{8} \cdot(1-\delta)^{3}$.

Integrating over $\sigma$ from 0 to 1 by using the Simpson rule with 500 divisions and using $\frac{1}{2 \pi}\left(\frac{3}{8}\right)^{2} m_{e} \alpha^{8}=52.3951 \mathrm{sec}^{-1}$, we obtain (all numerical coefficients below are in units of $\left.\sec ^{-1}\right)$

$$
\begin{aligned}
& d_{i}=d_{i 0}(1-\delta)(1-\delta+Z \delta)^{4}, \quad \text { for } i=1,3,5,7, \\
& d_{2}=(1-\delta)(1-\delta+Z \delta)^{2}\left[\left((1-\delta)^{3}+Z^{2} \delta^{3}\right) a+(1-\delta+Z \delta)\left((1-\delta)^{3}+Z \delta^{3}\right) a^{\prime}\right], \\
& d_{4}=d_{40}(1-\delta)(1-\delta+Z \delta)^{2}\left((1-\delta)^{3}+Z^{2} \delta^{3}\right) \\
& d_{6}=(1-\delta)(1-\delta+Z \delta)^{2}\left[\left((1-\delta)^{3}+Z^{2} \delta^{3}\right) b+\left((1-\delta)^{2}-Z \delta^{2}\right) b^{\prime}\right],
\end{aligned}
$$

with $a=-4.3990 \times 10^{-1}, a^{\prime}=-3.9877 \times 10^{-2} b=-2.8271 \times 10^{-1}, b^{\prime}=7.2080 \times 10^{-2}$. The dependence on $\delta$ in the coefficients $d_{1}$ through $d_{7}$ comes from the recoil effects in the two-photons phase space (66). In the infinite nuclear mass limit $M=\infty$ and $\delta=0$, the coefficients $d_{i}$ 's become $d_{i 0}$ given by

$$
\begin{aligned}
d_{10}=3.0860, & d_{20}=-4.7978 \times 10^{-1}, \\
d_{30}=-1.1573, & d_{40}=-2.8271 \times 10^{-1}, \\
d_{50}=3.4852 \times 10^{-1}, & d_{60}=-2.1063 \times 10^{-1}, \\
d_{70}=-6.1464 \times 10^{-1} . &
\end{aligned}
$$

For positronium, $Z=1$ and $\delta=1 / 2$, the corresponding coefficients are given by

$$
\begin{aligned}
d_{1}=1.5430, & d_{2}=-5.9972 \times 10^{-2}, \\
d_{3}=-5.7865 \times 10^{-1}, & d_{4}=d_{6}=-3.5339 \times 10^{-2} \\
d_{5}=1.7426 \times 10^{-2}, & d_{7}=-3.0732 \times 10^{-1} .
\end{aligned}
$$

Notice that the electric quadrupole moment vanishes for the positronium and all the $(Z \alpha)^{2}$ corrections in (69) arise from finite mass correction. It is not suprising that $d_{4}=d_{6}$ 
for the positronium if we remember that $h^{\prime}$ comes solely from the electric quadrupole transition and is zero in the present case.

\section{Acknowledgements}

We would like to thank C.K. Chow for collaboration in the initial stage of this work and comments on the manuscript. CKA acknowledges the hospitality of the Chinese University of Hong Kong through a C.N. Yang fellowship while on sabbatical leave from the University of South Carolina.

\section{References}

[1] C.K. Au, Phys. Lett. A 232 (1997) 162.

[2] G. Breit and E. Teller, Astrophys. J 91 (1940) 215.

[3] W.R. Johnson, Phy. Rev. Lett. 29 (1972) 1123.

[4] C.K. Au, Phys. Rev. A 32 (1985) 3734.

[5] M. Gavrila and A. Costescu, Phys. Rev. A 2 (1970) 1752.

[6] C.K. Au, J. Phys. B 11: Atom. Molec. Phys. (1978) 2781.

[7] S. Klarsfeld, Phys. Lett. A 30 (1969) 382.

[8] C.K. Au, Phys. Rev. A 14 (1976) 531. 